\title{
INFINITE FREE SET FOR SMALL MEASURE SET MAPPINGS
}

\author{
LUDOMIR NEWELSKI, JANUSZ PAWLIKOWSKI AND WITOLD SEREDYŃSKI
}

\begin{abstract}
A set $A \subset X$ is free for a function $F: X \rightarrow P(X)$ provided $x \notin F(y)$ for any distinct $x, y \in A$. We show that, if $F$ maps the reals into closed subsets of measure less than 1 , then there is an infinite free set for $F$. This solves Problem 38(B) of Erdös and Hajnal [EH].
\end{abstract}

A set $A \subset X$ is free for a function $F: X \rightarrow P(X)$ if $x \notin F(y)$ for any distinct $x, y \in A$. Erdös and Hajnal [EH, Problem 38(B)] asked about the size of a free set for $F$ mapping the reals into closed sets of measure less than 1. Gładysz [G] proved the following theorem. Suppose $X$ is a separable metric space and $\mu$ is a finite Borel measure which vanishes on points. Then, for any function $F: X \rightarrow$ closed subsets of $X$, a free pair exists provided for some measurable function $\nu: X \rightarrow R, \mu(F(x)) \leq \nu(x)$ for all $x \in X$, and

$$
\int_{X} \nu d \mu<\frac{1}{2}(\mu(X))^{2}
$$

We generalize this result and find conditions for the existence of a $k$-element free set, $2 \leq k \leq \omega$.

We first fix some terminology. $\chi_{B}$ is the characteristic function of a set $B$. For $A \subset X \times Y, x \in X, y \in Y$, let $A_{x}=\{z \in Y:(x, z) \in A\}, A^{y}=\{z \in X:(z, y) \in A\}$. A $\sigma$-finite measure space is a triple $(X, X, \mu)$, where $X$ is a set, $\chi$ is a $\sigma$-field of subsets of $X$, and $\mu$ is a $\sigma$-finite measure in $\chi$. If $\mu$ is a measure, then $\mu^{*}$ and $\mu_{*}$ are the outer and inner measures respectively. Let $(X, \chi, \mu)$ be a $\sigma$-finite measure space. For a nonnegative real valued function $f$, write $\overline{\int_{X}} f d \mu$ for the upper integral, i.e. for $\inf \left\{\int_{X} g d \mu: g\right.$ measurable and $\left.g \geq f\right\}$. If $Y \subset X$, by a classical result of Łoś and Marczewski $[\mathbf{E M}]$, we can define a measure $\mu \uparrow Y$ on the $\sigma$-field $X \uparrow Y=\{V \cap Y: V \in X\}$ by $(\mu \uparrow Y)(V \cap Y)=\mu^{*}(V \cap Y)$. Then, for $V \subset Y,\left(\mu\lceil Y)^{*}(V)=\mu^{*}(V)\right.$, and if $\mu^{*}(Y)<\infty,(\mu \uparrow Y)_{*}(V)=\mu^{*}(Y)-\mu^{*}(Y \backslash V)$. Moreover, for any nonnegative real valued function $f, \overline{\int_{Y}} f d(\mu \uparrow Y) \leq \overline{\int_{X}} f d \mu$.

Now we state a Fubini type lemma.

LEMMA. Let $(X, \mathcal{X}, \mu)$ be a $\sigma$-finite measure space. Let $Y$ be a second countable topological space, and let $\nu$ be a $\sigma$-finite measure in the $\sigma$-field generated by closed subsets of $Y$. Then, for any set $A \subset X \times Y$ with all sections $A_{x}$ closed,

$$
\bar{\int}_{Y} \mu_{*}\left(A^{y}\right) d \nu(y) \leq \bar{\int}_{X} \nu\left(A_{x}\right) d \mu(x) .
$$

Received by the editors January 6, 1986.

1980 Mathematics Subject Classification (1985 Revision). Primary 04A05, 04A20; Secondary 28A25.

Key words and phrases. Set mapping, free set, Fubini's theorem. 
ProOF. We do it for $\mu$ and $\nu$ finite. Let $U$ be a countable base of $Y$. Given $\varepsilon>0$, find $B \supset A$ such that for $x \in X, \nu\left(B_{x} \backslash A_{x}\right)<\varepsilon$ and $Y \backslash B_{x}$ is a finite union of some members of $\mathcal{U}$. Next find $Z \subset X$ and a finite $\mathcal{V} \subset \mathcal{U}$ such that $\mu^{*}(Z)>\mu(X)-\varepsilon$ and that for $z \in Z, Y \backslash B_{z}$ is a union of some members of $\mathcal{V}$.

Let $Z=\mathcal{X} \uparrow Z$ and $\vartheta=\mu \uparrow Z$. Let $A$ be the finite field generated by $\mathcal{V}$. If $S$ is an atom of $A$, then there is $R_{S}$ with $B^{y} \cap Z=R_{S}$ for all $y \in S$. Find $T_{S} \in Z$ with $T_{S} \subset R_{S}, \vartheta\left(T_{S}\right)=\vartheta_{*}\left(R_{S}\right)$. Let $C=\bigcup\left\{S \times T_{S}: S\right.$ an atom of $\left.A\right\}$. Then

$$
\begin{aligned}
\int_{Y} \vartheta_{*}\left(B^{y} \cap Z\right) d \nu(y) & =\int_{Y} \vartheta\left(C^{y}\right) d \nu(y)=\int_{Z} \nu\left(C_{z}\right) d \vartheta(z) \\
& \leq \overline{\int_{Z}} \nu\left(B_{z}\right) d \vartheta(z) \leq \int_{X} \nu\left(B_{x}\right) d \mu(x)
\end{aligned}
$$

Moreover, by $\vartheta_{*}\left(B^{y} \cap Z\right)=\mu^{*}(Z)-\mu^{*}\left(Z \backslash B^{y}\right) \geq \mu^{*}(Z)-\mu(X)+\mu(X)-\mu^{*}\left(X \backslash B^{y}\right) \geq$ $\mu_{*}\left(B^{y}\right)-\varepsilon$,

$$
\begin{aligned}
\int_{Y} \mu_{*}\left(B^{y}\right) d \nu(y) & \leq \varepsilon \cdot \nu(Y)+\int_{Y} \vartheta_{*}\left(B^{y} \cap Z\right) d \nu(y) \\
& \leq \varepsilon \cdot \nu(Y)+\int_{X} \nu\left(B_{x}\right) d \mu(x)
\end{aligned}
$$

As $\varepsilon$ was arbitrary, the Lemma follows.

THEOREM. Let $X$ be a second countable topological space, and let $X$ be the $\sigma$-field generated by closed subsets of $X$. Let $\mu$ be a $\sigma$-finite measure in $X$. Suppose that $F \subset X \times X$ is such that $F_{x}$ is closed for all $x \in X$. Consider the mapping $x \rightarrow F_{x}$.

(a) Suppose that $\mu(X)<\infty, 2 \leq k<\omega$, and for $x \in X$ and $B \subset X$ with $\mu^{*}(B)>0$, we have

$$
\mu(X)>(k-1)\left(\mu^{*}(\{x\})+\frac{2}{\mu^{*}(B)} \int_{X} \chi_{B}(t) \mu^{*}\left(F_{t} \cap B\right) d \mu(t)\right) .
$$

Then there is a k-element free set. Moreover, if $k=2$, it suffices to check (*) for $B=X$.

(b) There is an infinite free set provided (i) or (ii) holds.

(i) $\mu(X)=\infty$ and for some $C<\infty$, for all $x \in X, \mu\left(F_{x}\right)<C$.

(ii) For all $x \in X, \mu\left(F_{x} \cup\{x\}\right)=0$.

Proof. (a) By the Lemma,

$$
\int_{X} \mu_{*}\left(F^{t}\right) d \mu(t) \leq \int_{X} \mu\left(F_{t}\right) d \mu(t) .
$$

Hence,

$$
\int_{X}\left(\mu_{*}\left(F^{t}\right)+\mu\left(F_{t}\right)\right) d \mu(t) \leq 2 \int_{X} \mu\left(F_{t}\right) d \mu(t) .
$$

So, for some $x \in X$,

$$
\mu_{*}\left(F^{x}\right)+\mu\left(F_{x}\right) \leq \frac{2}{\mu(X)} \bar{\int}_{X} \mu\left(F_{t}\right) d \mu(t) .
$$


Let $Y=X \backslash\left(F^{x} \cup F_{x} \cup\{x\}\right)$. By $(*)$ and $(* *), \mu^{*}(Y)>0$. Hence $Y \neq \varnothing$ and to get a 2-element free set we can take any $y \in Y$. Then $y \notin F^{x} \cup F_{x} \cup\{x\}$, so $\{x, y\}$ is free. If we want more, we use induction.

By (*), for $y \in Y$ and $B \subset Y$ with $\mu^{*}(B)>0$,

$$
\mu(X)>(k-1)\left(\mu^{*}(\{y\})+\frac{2}{\mu^{*}(B)} \bar{\int}_{X} \chi_{B}(t) \mu^{*}\left(F_{t} \cap B\right) d \mu(t)\right) .
$$

Also

Hence

$$
\mu(X)>(k-1)\left(\mu^{*}(\{x\})+\frac{2}{\mu(X)} \bar{\int}_{X} \mu^{*}\left(F_{t}\right) d \mu(t)\right) .
$$

$$
\begin{aligned}
(k-2) \mu(X)+ & \mu(X) \\
> & (k-2)(k-1)\left(\mu^{*}(\{y\})+\frac{2}{\mu^{*}(B)} \int_{X} \chi_{B}(t) \mu^{*}\left(F_{t} \cap B\right) d \mu(t)\right) \\
& +(k-1)\left(\mu^{*}(\{x\})+\frac{2}{\mu(X)} \int_{X} \mu^{*}\left(F_{t}\right) d \mu(t)\right) .
\end{aligned}
$$

By $(* *)$, after division by $(k-1)$,

$$
\begin{aligned}
\mu^{*}(Y) & \geq \mu(X)-\left(\mu^{*}(\{x\})+\frac{2}{\mu(X)} \int_{X} \mu\left(F_{t}\right) d \mu(t)\right) \\
& >(k-2)\left(\mu^{*}(\{y\})+\frac{2}{\mu^{*}(B)} \int_{X} \chi_{B}(t) \mu^{*}\left(F_{t} \cap B\right) d \mu(t)\right) .
\end{aligned}
$$

Now set $Y=X \uparrow Y$ and $\nu=\mu \uparrow Y$. By the above argument,

$$
\nu(Y)>(k-2)\left(\nu^{*}(\{y\})+\frac{2}{\nu^{*}(B)} \int_{Y} \chi_{B}(t) \nu^{*}\left(F_{t} \cap B\right) d \nu(t)\right)
$$

for any $y \in Y$ and any $B \subset Y$ with $\nu^{*}(B)>0$. That is, (*) holds for $Y$, $\nu$, and $k-1$. So, by the induction hypothesis, there is a $(k-1)$-element free set in $Y$. Clearly $Y \cup\{x\}$ is a $k$-element free set.

(b) For (i) the key is the following claim.

Claim. There is $x \in X$ such that $\mu^{*}\left(X \backslash F^{x}\right)=\infty$.

Proof of Claim. Suppose not. Find $Y \subset X$ and $N<\omega$ such that $C<$ $\mu^{*}(Y)<\infty$ and $\mu^{*}\left(X \backslash F^{y}\right)<N$ for $y \in Y$. Then, for $B \in \mathcal{X}, \mu_{*}\left(F^{y} \cap B\right) \geq$ $\mu(B)-N$ for all $y \in Y$. Set $\mathcal{Y}=\mathcal{X} \uparrow Y$ and $\nu=\mu \uparrow Y$. By the Lemma, for any $B \in \mathcal{X}$ such that $0<\mu(B)<\infty$,

$$
\begin{aligned}
\mu(B) \cdot \mu^{*}(Y)-N \cdot \mu^{*}(Y) & \leq \int_{Y} \mu_{*}\left(F^{y} \cap B\right) d \nu(y) \\
& \leq \overline{\int_{B}} \nu\left(F_{x} \cap Y\right) d \mu(x) \leq \mu(B) \cdot C .
\end{aligned}
$$

So $C \geq \mu^{*}(Y) \cdot(\mu(B)-N) \cdot \mu(B)^{-1}$, which, for large $\mu(B)$, contradicts $C<\mu^{*}(Y)$. The Claim is proved. 
Now, pick $x \in X$ by the Claim, and look at $Y=X \backslash\left(F_{x} \cup F^{x} \cup\{x\}\right)$. Since $\mu^{*}(Y)=\infty$, we can apply the Claim to $Y, X \uparrow Y$, and $\mu \uparrow Y$. So pick $y \in Y$ by the Claim. Since $y \notin F_{x} \cup F^{x} \cup\{x\},\{x, y\}$ is free. Moreover,

$$
(\mu \uparrow Y)^{*}\left(Y \backslash\left(F_{y} \cup F^{y} \cup\{y\}\right)\right)=\infty,
$$

so we can continue and pick in this fashion an infinite free set.

For (ii) the key is that by (**) of (a), there is $x \in X$ such that $\mu_{*}\left(F^{x}\right)=0$, so $\mu^{*}\left(X \backslash\left(F_{x} \cup F^{x} \cup\{x\}\right)\right)=\mu(X)$. Now we can find an infinite free set in the fashion of (i).

COROLlaRY (1). On the real line consider a map $x \rightarrow F(x)$, where $F(x)$ is closed of measure less than 1 . Then there is an infinite free set.

This answers Problem 38(B) of Erdös and Hajnal [EH].

COROLlaRY (2). On the unit interval consider a map $x \rightarrow F(x)$, where $F(x)$ is closed of measure zero. Then there is an infinite free set.

In this case $F(x)$ is nowhere dense. Erdös [E] proved that such mappings have infinite free sets. But the above argument applies also to measures which do not force closed sets of measure zero to be nowhere dense.

COROLlary (3). Suppose that $X$ is a second countable topological space, $\mu$ is a measure in the $\sigma$-field generated by closed sets, and $\mu$ vanishes on points. Let $2 k-2<\mu(X)<\infty, k \geq 2$. Then, for any mapping $x \rightarrow F(x)$ with $F(x)$ closed of measure less than 1 , there is a $k$-element free set. In fact there are disjoint sets $B_{1}, \ldots, B_{k}$ of positive outer measure such that any selector $x_{1} \in B_{1}, \ldots, x_{k} \in B_{k}$ is free.

Proof. Let $\mathcal{U}$ be a countable base of $X$ and let $\varepsilon>0$ be small. Find $A$ such that $A_{x} \supset F(x), \mu\left(A_{x} \backslash F(x)\right)<\varepsilon$, and $X \backslash A_{x}$ is a finite union of members of $\mathcal{U}$. Next take $Y \subset X$ with $\mu^{*}(Y)>\mu(X)-\varepsilon$, and $\mathcal{V} \subset \mathcal{U}$ such that for all $y \in Y$, $A_{y}$ is a union of elements of $\mathcal{V}$. By the Lemma (the proof) there is $S \subset Y$ with $\mu^{*}(S)>0$, and $R_{S} \subset Y$ with $\mu^{*}\left(R_{S}\right)<1$, such that for all $y \in S, A^{y} \subset R_{S}$. Refine $S$ further to get $T \subset S$ with $\varepsilon>\mu^{*}(T)>0$, and $P_{T}$ such that for all $y \in T$, $A_{y}=P_{T}$. Then set $B_{1}=T$ and look at $Y \backslash\left(R_{S} \cup T \cup P_{T}\right)$. If $\varepsilon$ is small, this set has outer measure greater then $2(k-1)-2$ and we can continue.

Corollary (4). Suppose that $F:\{0,1, \ldots, n-1\} \rightarrow P(\{0,1, \ldots, n-1\})$, $|F(x)| \leq m$ for all $x$. Then there is a k-element free set iff $k<1+n /(2 m+1)$.

Proof. For the "if" direction use Theorem (a) with the counting measure. For the "only if" direction we give the following example. Let $n=(2 m+1) d+r$, $0<r \leq 2 m+1$. For $i=0, \ldots, d-1,0 \leq c<2 m-1$, let

$$
F((2 m+1) i+c)=\left\{(2 m+1) i+(c+j)_{\bmod (2 m+1)}: j=c+1, \ldots, c+m\right\},
$$

and, for $0 \leq c<r$, let

$$
F((2 m+1) d+c)=\left\{(2 m+1) d+(c+j)_{\bmod (r)}: j=c+1, \ldots, c+m\right\} .
$$

Then always $|F(x)| \leq m$, and any free set for $F$ has at most one element in each segment $(2 m+1) i \leq x<(2 m+1)(i+1)$. The number of segments is $d+1$, so there is no free set of size $\geq 1+d+1 \geq 1+n /(2 m+1)$. 
NOTES. (1) Corollary (4) shows that the assumptions of the Theorem are (in some sense) essential.

(2) It seems worthwhile to note the following corollary of the Lemma. Let $\lambda$ be the Lebesgue measure. Suppose $A$ is a subset of the plane with all vertical sections closed of measure zero. Then

$$
\lambda^{*}\left(\left\{y: \lambda_{*}\left(A_{y}\right)>0\right\}\right)=0 .
$$

(3) Topology is not necessary. Let $(X, X, \mu)$ be a $\sigma$-finite measure space. Let $\subset \subset X$ be a countable field. For $A \subset X$ set $\bar{\mu}(A)=\inf \{\mu(C): C \in C$ and $A \subset C\}$. Then we can drop the assumption that $F_{x}$ is closed but we must replace $\mu\left(F_{x}\right)$ by $\bar{\mu}\left(F_{x}\right)$. This is an equivalent approach because $C$ can be taken as a base for the topology on $X$.

ACKNOWLedgment. We are grateful to Professor Wegglorz for drawing our attention to the Erdös-Hajnal problem.

ADDED IN PROOF. 1. D. H. Fremlin remarks that if $\mu$ is atomless (i.e. $\mu^{*}(\{x\})$ $=0$ for each $x \in X)$, then the condition $(*)$ in Theorem (a) can be weakened to $(\mu(X))^{2}>2(k-1) \underline{\int} \mu\left(F_{x}\right) d \mu(x), \underline{\int}$ being the lower integral. The argument is based on the equality

$$
\int_{X} \mu\left(F_{x}\right) d \mu(x)=\inf \left\{\left(\nu^{2}\right)^{*}(F): \nu \text {-a measure on } X \text { extending } \mu\right\}
$$

and the following fact: if $E \subset X^{2}$ is $\nu^{2}$-measurable with $(k-1) \nu^{2}(E)<(\nu(X))^{2}$ then there are points $x_{1}, \ldots, x_{n} \in X$ such that $\left(x_{i}, x_{j}\right) \notin E$ whenever $i<j<n$.

2. In a forthcoming paper, Half Fubini theorem, the second author discusses the following strenghtened version of the Lemma:

If $f:[0,1] \times[0,1] \rightarrow[0,1]$ then $\bar{\int}^{R}\left(\underline{\int}^{L} f(x, y) d x\right) d y \leq \underline{\int}^{L}\left(\bar{\int}^{R} f(x, y) d y\right) d x, L$ standing for Lebesgue, $R$-for Riemann integral.

\section{REFERENCES}

[E] P. Erdös, Some remarks on set theory. III, Michigan Math. J. 2 (1953-54), 51-57.

[EH] P. Erdös and A. Hajnal, Unsolved problems in set theory, Axiomatic Set Theory, Proc. Sympos. Pure Math., Vol. 13, Part 1, Amer. Math. Soc., Providence, R.I., 1971, pp. 17-48.

[G] S. Gładysz, Bemerkungen über die Unabhangigkeit der Punkte in Bezug auf Mengenwertigen Funktionen, Acta Math. Acad. Sci. Hungar. 13 (1962), 199-201.

[EM] J. Loś and E. Marczewski, Eatensions of measure, Fund. Math. 36 (1949), 267-276.

Instytut Matematyczny, Polska AKademia NaUk, Ul. Kopernika 18, WrocŁaW, POLAND (Current address of Ludomir Newelski and Witold Seredyński)

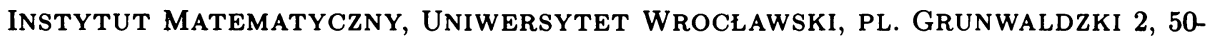
384 WrocŁaW, POLAND (Current address of Janusz Pawlikowski) 